\title{
The Unique Capabilities of Auger Electron Spectroscopy
}

\author{
R.A. Price ${ }^{1}$, J.F. Moulder ${ }^{1}$, D.F. Paul ${ }^{1}$, J.S. Hammond ${ }^{1}$ \\ 1. Physical Electronics, 18725 Lake Drive East, Chanhassen, MN 55317, USA
}

While the focus of this meeting is on instrumentation with very high lateral spatial resolution Physical Electronics specializes in instrumentation with ultra-shallow surface sensitivity. The performance characteristics of many materials systems are strongly linked to surface composition. Modified surface layers may be included by design to enhance performance, or they may occur unknowingly and create a complex problem to resolve. Having instrumentation in your laboratory with nano-scale surface sensitivity may be crucial to accelerating the development of new materials and products that have performance characteristics dependent on surface, thin film, or interface composition. We will present application examples that highlight the unique capabilities of Auger Electron Spectroscopy (AES).

AES instruments us a finely focused electron beam to excite a samples surface and cause among other things Auger electrons to be ejected from a sample. Due to the low energy of the Auger electrons (0$2000 \mathrm{eV}$ ) their escape depth is less than $10 \mathrm{~nm}$ making AES a surface analysis technique. With the exception of $\mathrm{H}$ and $\mathrm{He}$, it is possible to detect nearly all elements in the periodic table with detection limits that are approximately 0.1 atom percent of the ultra-small analysis volume. Elemental and in many cases chemical state information is obtainable based on the energy and shape of peaks in Auger spectra. The ultimate chemical spatial resolution for AES instruments is in the 6-8 $\mathrm{nm}$ range.

A common metallurgical application for surface analysis instruments is the ability to detect ultra-thin layers of tramp elements that may segregate to a grain boundary surface and cause embrittlement. Figure 1 is a secondary electron image of a steel fracture surface. Figure 2 shows an AES spectrum from the fracture surface showing the presence of antimony $(\mathrm{Sb})$ at the grain boundary surface. While $\mathrm{Sb}$ is present in the PPM range in the bulk of the steel the AES spectra show the presence of an atomically thin grain boundary layer that contains several atom percent of Sb. This information is readily accessible with AES but impossible to detect by other probe beam based analysis methods which probe a much larger analysis volume.

In addition to the ability to observe ultra-thin layers, AES provides the ability to obtain compositional information from nanoscale particles that are difficult to characterize by other probe beam based analysis methods such as SEM-EDS which have a much larger analysis volume. In this presentation we will present metallurgical, semiconductor and nanomaterial AES application examples that show the ability to characterize thin layers, ultra small features, and ultra-thin films.

Physical Electronics also develops and manufactures X-ray Photoelectron Spectroscopy (XPS) and Time-of-Flight Secondary Ion Mass Spectrometry instruments for surface analysis applications. The capabilities of these surface analysis techniques is summarized in Table 1 on the following page. 


\section{Comparison of Surface Analysis Techniques}

\begin{tabular}{|c|c|c|c|}
\hline & AES & XPS & TOF-SIMS \\
\hline Probe Beam & Electrons & Photons & Ions \\
\hline Analysis Beam & Electrons & Electrons & Ions \\
\hline Spatial Resolution & $0.008 \mu \mathrm{m}$ & $7.5 \mu \mathrm{m}$ & $0.10 \mu \mathrm{m}$ \\
\hline Sampling Depth $(\AA)$ & $5-75$ & $5-75$ & $1-10$ \\
\hline Detection Limits & 0.1 atom $\%$ & 0.05 atom $\%$ & $1 \mathrm{ppm}$ \\
\hline Information Content & $\begin{array}{l}\text { Elemental } \\
\text { Chemical }\end{array}$ & $\begin{array}{l}\text { Elemental } \\
\text { Chemical }\end{array}$ & $\begin{array}{l}\text { Elemental } \\
\text { Chemical } \\
\text { Molecular }\end{array}$ \\
\hline Quantification & Good & Excellent & Std. needed \\
\hline
\end{tabular}

Table 1. Comparison of surface analysis techniques

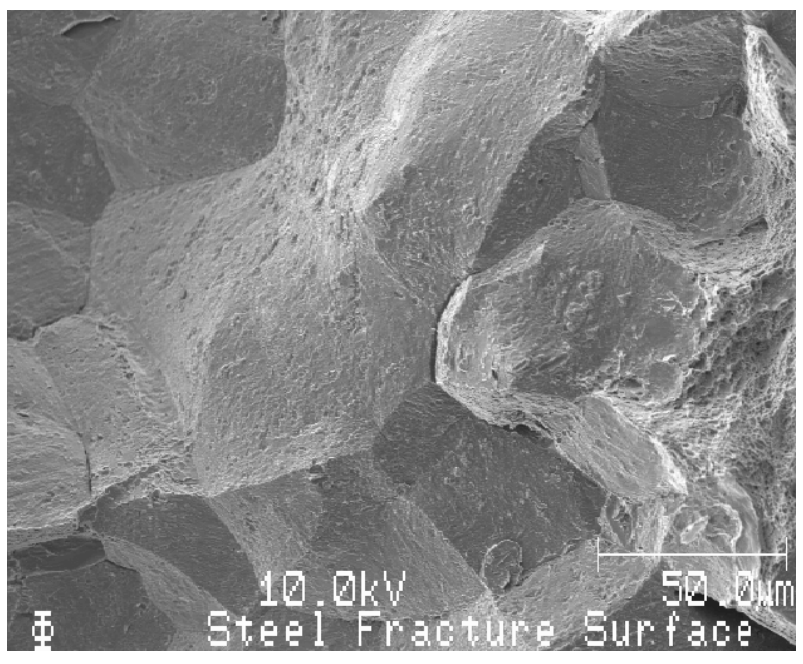

Figure 1. SE image of a steel fracture surface.

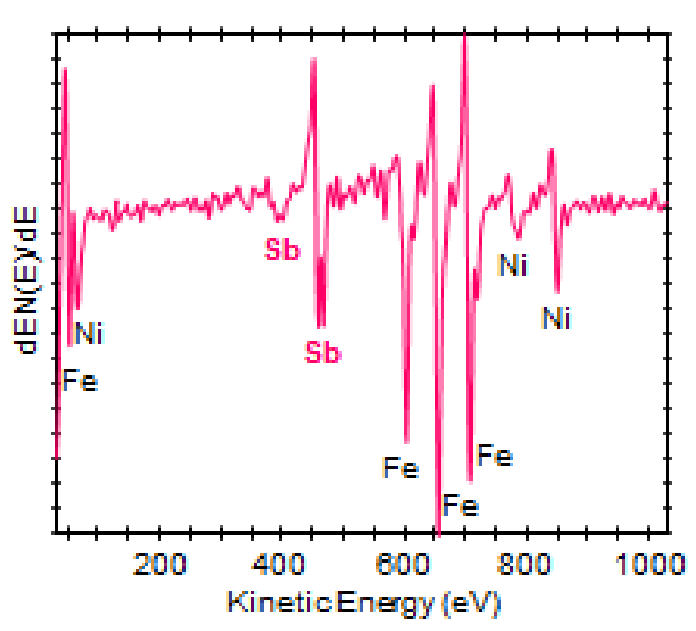

Figure 2. AES spectrum from the steel fracture surface showing $\mathrm{Sb}$ segregation to the grain boundary surface. 